\title{
Postpartum pain due to thrombosed varicose veins of the round ligament of the uterus
}

\author{
Mohammed S. Al-Qudah
}

Department of Surgery, Faculty of Medicine, University of Jordan, Jordan University Hospital, Amman, Jordan

\begin{abstract}
Summary: Postpartum thrombosis of varicose veins of the round ligament may present in a clinical picture similar to an irreducible inguinal hernia. Two such unusual cases are reported. One patient underwent surgery while the other, with a presumptive diagnosis of the same condition, was observed, with no complications occurring in both patients. This condition has not to my knowledge been previously reported. The increased incidence of both varicose veins and thrombosis in the postpartum period should alert the physician to the diagnosis of such conditions.
\end{abstract}

\section{Introduction}

Both varicose veins and thrombosis are known to occur with an increased frequency in pregnancy and puerperium. This combination usually manifests as superficial thrombophlebitis or deep vein thrombosis of the lower limbs and less commonly the pelvic veins. Postpartum thrombosis of varicose veins of the round ligament has not to our knowledge been previously reported.

\section{Case report}

\section{Case 1}

A 27 year old woman developed severe pain in the left inguinal region 24 hours after a normal vaginal delivery. She had no bowel symptoms or abdominal colic. Her vital signs were normal. Abdominal examination disclosed a firm and tender mass in the left inguinal region medial to the pubic tubercle, averaging $2.5 \mathrm{~cm}$ in diameter. The mass had no cough impulse and was irreducible. A plain film of the abdomen was normal. The diagnosis of an irreducible inguinal hernia was made. At emergency operation, the round ligament was oedematous, congested and contained thrombosed varicose veins. Exploration did not reveal a hernial sac. This segment was excised (Figure 1). The histopathology confirmed the diagnosis of thrombosed varicose veins of the round ligament. The postoperative

Correspondence: M.S. Al-Qudah, M.D., F.R.C.S.(Ed.), PO Box 6593, Amman, Jordan.

Accepted: 19 February 1993 course was uneventful. At follow up visit one month later, she had no problems.

\section{Case 2}

A 29 year old woman on her third postpartum da was referred from the gynaecologist with a diagnosis of an irreducible left inguinal hernia, after complaining of sudden pain in that region. On examination she had a tender and firm mass $2 \mathrm{~cm}$ in diameter in the left groin. However a firm cord $\bar{c}$ could be felt extending down to the labium which itself was free of varicosities. Otherwise, the physical examination was normal. The patient was

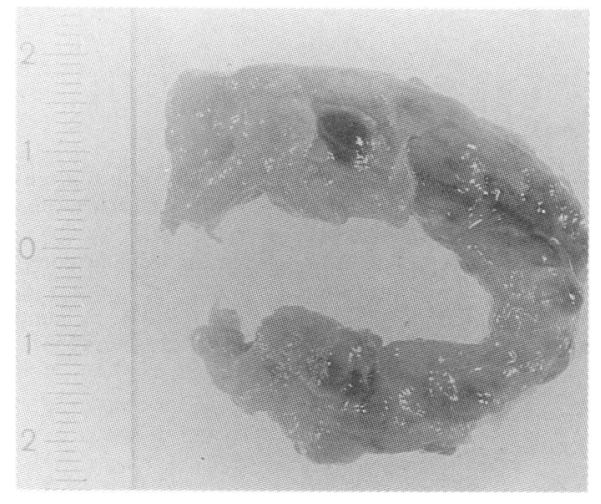

Figure 1 The excised part of the round ligament in case 1. The ligament is thickened with evident thrombosed varicose veins. 
observed and followed up in the outpatient clinic, and the mass diminished in size over the following week with the disappearance of pain and no hernias or masses detectable in the groin.

\section{Discussion}

Phlebothrombosis has an increased incidence during pregnancy and in the immediate postpartum period. The cause has been attributed to decreased venous flow, the result of mechanical pressure of the gravid uterus on the intra-abdominal veins. ${ }^{1}$ During labour this pressure is at its peak, the engaged fetal head being responsible. ${ }^{2} \mathrm{~A}$ decreased venous tone presumably due to oestrogen and progesterone also contributes to venous stasis. ${ }^{3} \mathrm{~A}$ hypercoagulable state exists during pregnancy, but in labour it reaches its maximum. ${ }^{4-6}$

The round ligament commences at the lateral angle of the uterus and is around $10-12 \mathrm{~cm}$ long. It contains veins, branches from the ovarian artery, lymphatics and nerves. Distally it breaks into several strands that are inserted into the areolar tissue of the labium majus. However, sometimes the ligament does not reach the labium and ends by fusing with its coverings which correspond to those found in the spermatic cord. In a postmortem dissection of ten females, none of the round ligaments reached the labium.?

The presentations of uncomplicated genital varicose veins include the syndrome of pelvic congestion, ${ }^{8}$ abdominal masses and inguinal masses. When complicated by rupture or thrombosis they stimulate common obstetric emergencies, ${ }^{9,10}$ and when the ovarian vein is involved a picture of acute abdomen can result. ${ }^{11}$ However, Borrero reported a case of spontaneous haemorrhage into the round ligament that presented with a sudden painful mass in the groin together with uterine bleeding, and he also operated on the patient with a preoperative diagnosis of an incarcerated inguinal hernia. ${ }^{12}$

In the first case, the mass was felt in the course of an indirect inguinal hernia just medial to the pubic tubercle and the likelihood of an irreducible inguinal hernia could not be ruled out. If labial varicose veins are involved, one would then see obvious varicose veins in the labia or the ligament may be felt as an indurated cord extending into the labium itself as in the second case described. However, if this is not the case then differentiation from other causes of acute painful groin masses may be impossible. The cause for this may be due to the anatomy of the round ligament failing to extend down to the labium.

It seems most probable that this condition is more common than the absence of reports would suggest, because of the high incidence of both postpartum phlebothrombosis and varicose veins of pregnancy. It is obvious that these manifestations can be extremely difficult to separate from the more common surgical causes and it is unlikely that the diagnosis can be made solely on clinical grounds. The use of ultrasound has shown some encouraging results for diagnosis of pelvic varicosities as described by Frede. ${ }^{13}$ Computed tomography and ultrasonography had been utilized successfully in the preoperative diagnosis of thrombosed ovarian veins. " The diagnosis of thrombosis of varicose veins of the round ligament should be entertained in females in the postpartum period who present with painful and tender inguinal swellings. A high degree of suspicion, possibly coupled with an accurate diagnostic modality that still needs to be established, may help avoid unnecessary surgery for such conditions.

\section{References}

1. Wright, H.P., Osborn, S.B. \& Edmonds, D.G. Changes in the rate of flow of venous blood in the leg during pregnancy measured with radioactive sodium. Surg Gynecol Obstet 1950, 90: $481-484$.

2. Coon, W.W. Epidemiology of venous thromboembolism Ann Surg 1977, 186: 149-164.

3. Flessa, H.C., Glueck, H.I. \& Dritschilo, A. Thromboembolic disorders in pregnancy: pathophysiology, diagnosis, and treatment, with emphasis on heparin. Clin Obstet Gynecol 1974, 17: 195-235.

4. Bonnar, J., McNicol, G.P. \& Douglas, A.S. Coagulation and fibrinolytic mechanisms during and after normal childbirth. Br Med J 1970, 2: 200-203.

5. Bonnar, J., McNicol, G.P. \& Douglas, A.S. The blood coagulation and fibrinolytic systems in the newborn and the mother at birth. J Obstet Gynecol Br Commonw 1971, 78: $355-360$

6. Burstein, R., Alkjaersig, N. \& Fletcher, A. Thromboembolism during pregnancy and the postpartum state. $J$ Lab Clin Med 1971, 78: 838.

7. Attah, A.A. \& Hutson, J.M. The anatomy of the female gubernaculum is different from the male. Austral N Z Surg 1991, 61: $380-384$.

8. Hanzlik, H. \& Leissering, J.C. Parametrial phlebectasia: A clinical-pathologic entity. Am J Obstet Gynecol 1976, 125: 431-434.

9. Georgakopoulos, P.A. Perinatal rupture of genital varicosities. Int Sur 1979, 64: 74-75.

10. Palatynski, A. \& Zdziennicki, A. Spontaneous rupture of uterine varicose veins during labor; simulating premature separation of the placenta. Zentralbl Gynakol 1985, 107: 54-57.

11. Loos, W., von Hugo, R., Rath, W. et al. Puerperal ovarian vein thrombophlebitis. Geburtshlfe Frauenheilkd 1988, 48: $483-488$.

12. Borrero, E., Weil, P.H. \& Cooper, J.J. Haemorrhage into the round ligament. JAMA 1983, 249: 3006.

13. Frede, T.E. Ultrasonic visualization of varicosities in the female genital tract. J Ultrasound Med 1984, 3: 365-369. 